\title{
Dynamic Methanation of By-product Gases from Integrated Steelworks
}

\author{
P. Wolf-Zoellner ${ }^{1 *}$, A. R. Medved ${ }^{1}$, A. Krammer ${ }^{1}$, K. Salbrechter ${ }^{1}$, M. Lehner ${ }^{1}$ \\ 1. Chair of Process Technology and Industrial Environmental Protection, Montanuniversität Leoben, Austria, \\ *corresponding author: philipp.wolf-zoellner@unileoben.ac.at
}

\section{Introduction and Motivation:}

Integrated steelworks are major contributors to global emissions of carbon sources such as carbon dioxide $\left(\mathrm{CO}_{2}\right)$. About 27 to $30 \%$ of any industrial $\mathrm{CO}_{2-}$ emissions result from such integrated steelworks. [1, 2] In order to achieve the challenging goals of the Paris climate agreement it is key to work on ways to rigorously reduce these $\mathrm{CO}_{2}$-emissions and to incorporate green energy source in the steelmaking process.

The by-product gases accumulating in a steelworks plant, such as the blast furnace gas (BFG), basic oxygen furnace gas (BOFG) and coke oven gas (COG) have a very rich content of $\mathrm{CO}_{2}$ and $\mathrm{CO}$ (carbon monoxide). At the current stage, these byproduct gases are stored and utilized internally, but additional fossil energy sources are needed to cover the whole energy demand for any auxiliary energy conversion. (Fig. 1) [3]

One way of re-using certain gas streams and substitute the need of fossil fuels would be through synthesis processes like methanation where $\mathrm{CO}_{2}$ and $\mathrm{CO}$ react with hydrogen $\left(\mathrm{H}_{2}\right)$, gathered from green energy sources, to methane $\left(\mathrm{CH}_{4}\right)$ and vapor.

Although these reactions are well-known, the kinetics and behavior under dynamic and transient conditions are yet to be investigated. This is especially important for steelworks processes, as the concentrations and the total volume flow of the by-product gases vary frequently based on to the related operational working step. Even more, the amount of available hydrogen required for the methanation synthesis is only dynamically available too. It is based on an economically driven electrolyzing unit and the electricity price consequently.

The reaction behavior of a dynamically driven power to gas plant needs to be investigated to provide a fundamental base for its implementation in an integrated steelworks plant.

\section{Methodology and Experiments}

The above-mentioned investigation is carried out within the EU-funded project "i3upgrade". For the necessary experiments under dynamic conditions, the existing pilot rig at the Chair of Process Technology and Industrial Environmental Protection at the Montanuniversitaet Leoben is used, which consists of three reactors, each filled with a commercial Nibased bulk catalyst, or $\mathrm{Ni} / \mathrm{Al}_{2} \mathrm{O}_{3}$ washcoated honeycomb catalysts. (Fig. 2) [4]

A series of experiments with synthetic BFG and BOFG has been carried out. To achieve the reaction goal of $100 \% \mathrm{CO}_{x}$ conversion, an optimum hydrogen excess rate to stoichiometry was evaluated and applied for dynamic situations as they occur in an integrated steelworks plant. This includes variations in total volume flow of by-product gases, hydrogen 
availability as well as dynamic cycle frequency and duration.

\section{Conclusion and Outlook}

The presentation will contain broad results of lab-scaled methanation experiments conducted under steady state and dynamic conditions representing the cases from an integrated steelwork. The influences on the synthesis, reactor behavior as well as the degradation of the catalyst due to the frequent load changes will be addressed. Furthermore, the performance of the commercial bulk catalyst will be compared with the newly developed wash-coated honeycombs.

\section{References}

[1] A review of thermochemical processes and technologies to use steelworks offgases, W. Uribe-Soto et al., Renewable and Sustainable Energy Reviews 74 (2017), pp. 809-823

[2] A. Hasanbeigi, 2017, URL: https://www.globalefficiencyintel.com /new-blog/2017/nfographic-steelindustry-energy-emissions

[3] Hauser, A. "i3upgrade - intelligent integrated industries". URL:

https://www.i3upgrade.eu, 02.04.2019.

[4] Biegger, P. „Keramische Wabenkatalysatoren zur chemischen Methanisierung von $\mathrm{CO} 2$ ". $\mathrm{PhD}$ thesis; Austria, 2017.

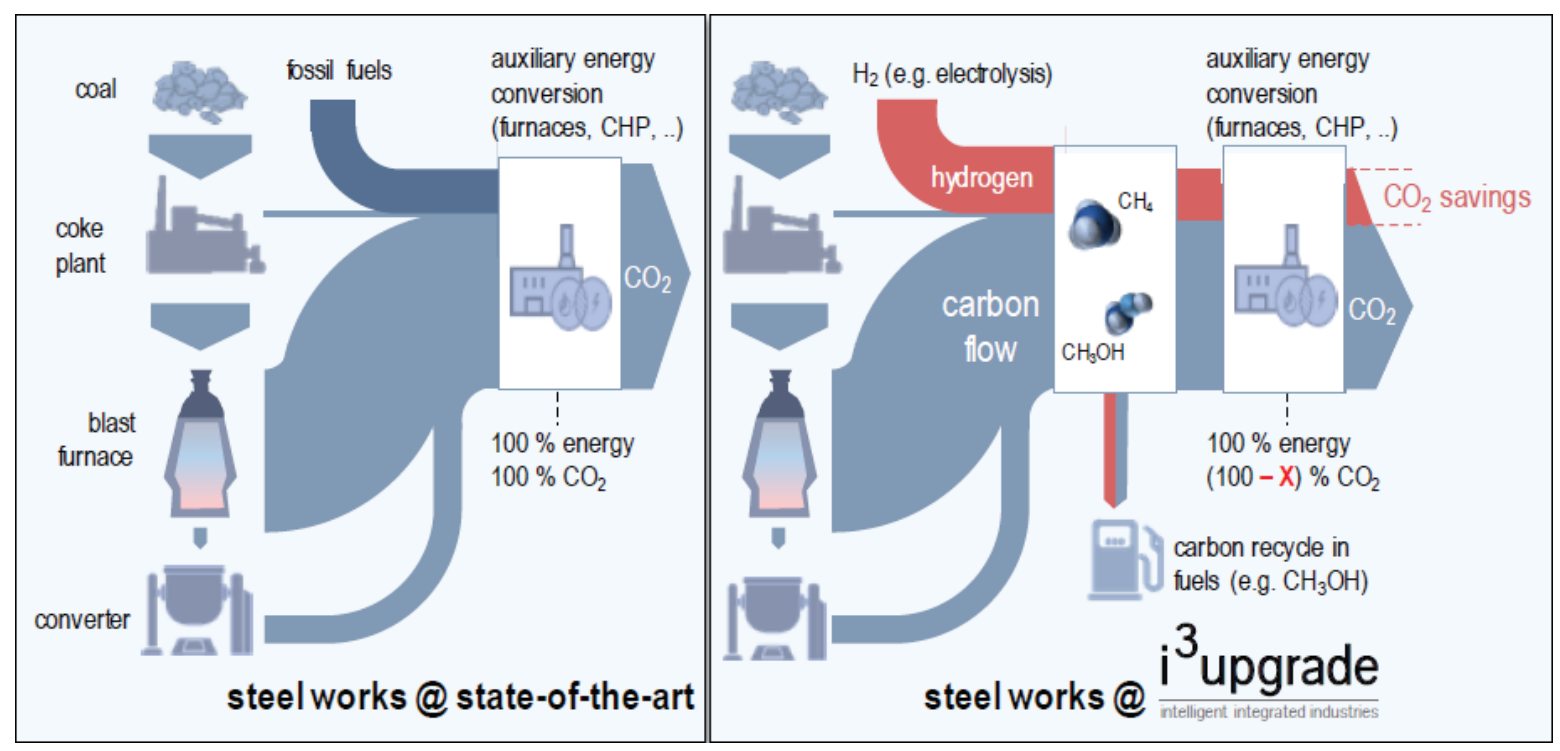

Fig. 1: Upgraded carbon flow in an integrated steelworks plant as anticipated with i3upgrade

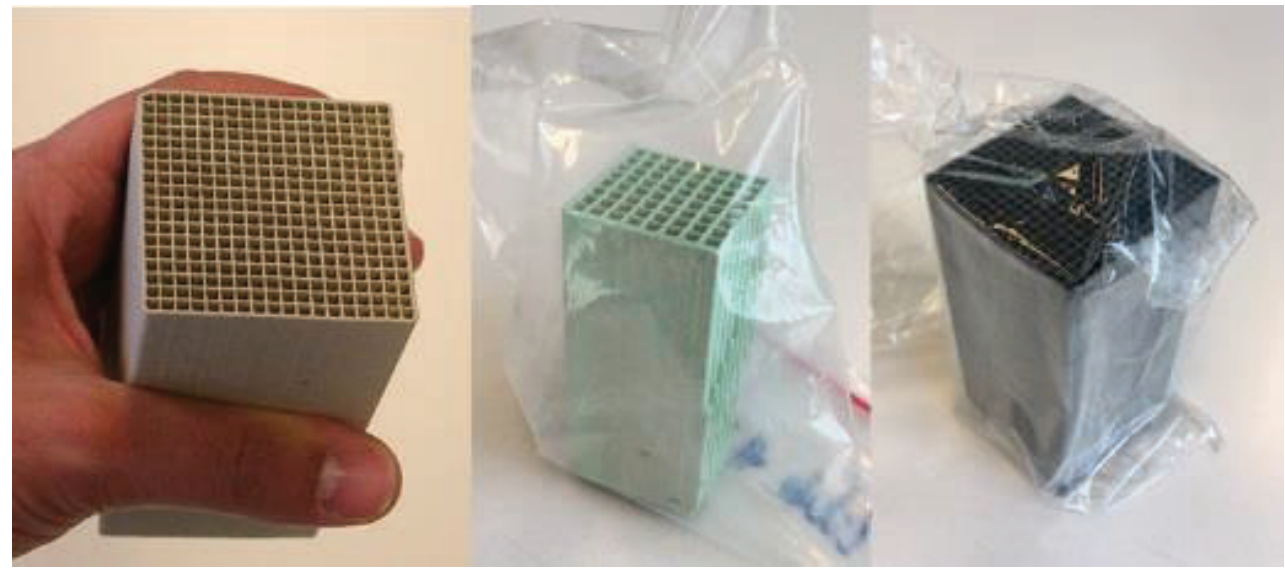

Fig. 2: Honeycomb monoliths (from left to right: raw, wash-coated, used) 